\title{
Sequência de atividades didáticas utilizando espirógrafo
}

\author{
Fernando de Oliveira Cezarino (1) Marcelo Firer (1)
}

\begin{abstract}
Resumo
Este texto apresenta uma sequência de atividades didáticas para ser realizada com alunos do Ensino Básico, com o objetivo de explorar o funcionamento do brinquedo chamado espirógrafo. Os conteúdos matemáticos utilizados e explorados são elementares (proporcionalidade, mínimo múltiplo comum e máximo divisor comum), e a sequência visa propiciar aos alunos uma descoberta orientada, baseada em observações de fenômenos, e concluída com uma demonstração transformacional.
\end{abstract}

Palavras-chave: espirógrafo; descoberta orientada; demonstração transformacional

\begin{abstract}
This text presents a sequence of teaching activities to be performed with basic education students, with the objective of exploring the working of a toy called spirograph. The mathematical subjects utilized and explored are elementary (proportionality, least common multiple and greatest common divisor) and the sequence aims to provide to the students a guided discovery, based on observations of phenomena, and concluded with a transformational proof.
\end{abstract}

Keywords: spirograph; guided discovery; transformational proof

\section{Introdução}

Um espirógrafo é um brinquedo formado por um conjunto de engrenagens que podem ser utilizadas duas de cada vez, uma fixa e outra móvel girando em torno da fixa. Na engrenagem móvel há vários orifícios onde pode ser inserida a ponta de uma caneta ou de um lápis a fim de desenhar o caminho percorrido pelo orifício. O design mais popular atualmente do brinquedo foi inventado pelo engenheiro britânico Denys Fisher [2].

O desenho traçado por um ponto fixo em uma curva rolando sem deslizar sobre outra curva é chamado de rolete [17]. As curvas traçadas por espirógrafos onde as duas engrenagens são circulares são tipos especiais de roletes: hipotrocoides (caso a engrenagem móvel esteja dentro da fixa) ou epitrocoides (caso a engrenagem móvel esteja fora da fixa). A Figura 1 mostra exemplos de desenhos que podem ser obtidos com um espirógrafo.

O programa "Isto é Matemática" [1] tem um episódio dedicado ao espirógrafo, que pode ser visto no YouTube em <https://youtu.be/ieVefMuW2jA>. Além disso, no próprio YouTube, inúmeros vídeos de utilização de espirógrafo podem ser encontrados. Por exemplo, os vídeos em <https://youtu. 

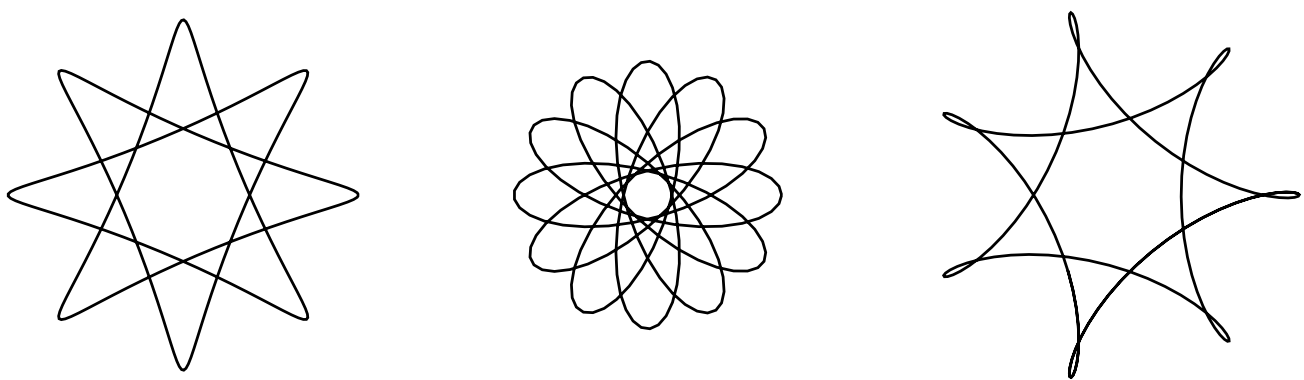

Figura 1: Exemplos de hipotrocoides obtidos com um espirógrafo.

be/gRo_FLWprGc> e <https://youtu.be/dzZ5bBslXdw> mostram o desenho de diferentes padrões que podem ser obtidos com um espirógrafo moderno. Outro recurso on-line interessante é o site Inspiral $W e b[8]$, onde se encontra uma simulação digital de um espirógrafo.

Existem diversas propostas de atividades utilizando o espirógrafo, a grande maioria considerando a parametrização das curvas, destinadas principalmente para alunos no final do Ensino Médio. Neste texto será descrita uma proposta de sequência de atividades destinadas a alunos dos anos finais do Ensino Fundamental, investigando propriedades das hipotrocoides utilizando conceitos como de mínimo múltiplo comum e proporcionalidade. Os espirógrafos mais baratos permitem traçar apenas hipotrocoides, e por esse motivo serão exploradas apenas essas curvas, embora seja possível facilmente estender todo o estudo para epitrocoides.

Do ponto de vista pedagógico, esta sequência de atividades pode ser classificada como aprendizagem por descoberta, um método indutivo de ensino em que os estudantes descobrem os conteúdos por eles mesmos, cabendo ao professor prover algum grau de instrução ou orientação no decorrer no processo de aprendizagem [15]. Apesar de a matemática ser uma ciência dedutiva, o raciocínio indutivo é importante para formular conjecturas e fazer descobertas (conforme reconhecido por George Pólya, no seu mais famoso livro [16]). Em última instância, o objetivo é orientar a descoberta dos alunos sobre estruturas matemáticas que permitam um conhecimento preditivo sobre o espirógrafo: a possibilidade de determinar diversas características do desenho a partir apenas da escolha inicial de engrenagens e orifício da engrenagem móvel.

Este texto está baseado em uma dissertação de mestrado que tem como foco uma sequência desenvolvida para explorar a matemática do espirógrafo.

Sobre a organização do texto, na Seção 2, são estabelecidas a notação e as definições matemáticas pertinentes ao problema. Na Seção 3 a sequência de atividades é contextualizada pedagógica e matematicamente dentro da variedade de métodos indutivos de ensino e aprendizagem e de tipos de demonstração. Finalmente, na Seção 4, é apresentada a estrutura conceitual da sequência e uma breve descrição de cada uma das onze atividades, além de uma delas explicada com mais detalhes.

\section{A matemática do espirógrafo}

Uma hipotrocoide pode ser obtida por um ponto fixo em uma circunferência interna com raio $\mathrm{r}$ rolando sem deslizar em torno de uma circunferência externa com raio $\mathrm{R}>\mathrm{r}$. Considere o centro 
da circunferência externa coincidindo com o centro de um sistema cartesiano e uma cúspide ${ }^{1}$ da hipotrocoide sobre o eixo horizontal. Considere também que a circunferência interna move-se no sentido anti-horário e que $\theta$ é a medida do ângulo formado pelo eixo horizontal do plano cartesiano e o segmento que liga a origem ao centro da circunferência interna. Podemos então descrever a hipotrocoide em função de $\theta$ pelas equações:

$$
\mathrm{x}(\theta)=(\mathrm{R}-\mathrm{r}) \cos \theta+\rho \cos \left(\frac{\mathrm{R}-\mathrm{r}}{\mathrm{r}}\right) \theta \quad \text { e } \quad \mathrm{y}(\theta)=(\mathrm{R}-\mathrm{r}) \sin \theta-\rho \sin \left(\frac{\mathrm{R}-\mathrm{r}}{\mathrm{r}}\right) \theta
$$

onde $\rho$ representa a distância entre o centro da circunferência interna e o ponto considerado para traçar a curva. Detalhes da obtenção dessas equações podem ser encontrados, por exemplo, em [12]. Para mais informações sobre roletes e espirógrafos ver, por exemplo, [7].

As equações possibilitam um conhecimento completo da hipotrocoide: se pensarmos no ângulo $\theta(\mathrm{t})$ como uma função do tempo t, elas descrevem a posição da caneta ou do lápis a cada momento. Elas descrevem qualitativamente e quantitativamente a curva. Essa abordagem já foi utilizada ao se explorar o espirógrafo como recurso didático em, por exemplo, [11].

Neste trabalho é proposta uma sequência de atividades sem parametrização das curvas, focando em conteúdos contemplado nos currículos de Ensino Fundamental.

\subsection{Uma abordagem discreta do espirógrafo}

Pensando no espirógrafo físico, as circunferências interna e externa são representadas por engrenagens. A Figura 2 mostra essa representação, feita a partir da referência [8], com R, r e $\rho$ indicados. A Figura 3 mostra a hipotrocoide obtida com a configuração de engrenagens da Figura 2 em diferentes estágios da sua construção (cada imagem representa uma volta completa da engrenagem interna em torno da externa).

O ponto de partida é observar que, como todos os dentes têm o mesmo tamanho, o número de dentes das engrenagens é diretamente proporcional ao comprimento das circunferências e, consequentemente, aos seus raios. É possível então falar do número de dentes das engrenagens em vez de raio das circunferências. Trata-se de uma abordagem discreta, baseada no número de dentes, sempre um número inteiro. Isso possibilita um estudo dos desenhos obtidos com um espirógrafo (hipotrocoides) levando em conta aspectos qualitativos. A falta de uma parametrização impossibilita a predição de alguns aspectos quantitativos (o comprimento da curva, por exemplo), mas preserva aspectos qualitativos interessantes. Se perde algo em sua capacidade preditiva, a abordagem discreta carrega vantagens significativas para o ensino, pois não demanda a parametrização de curvas (como feito no início da presente Seção) e envolve apenas conteúdos constantes do programa de ensino das escolas do Ensino Fundamental².

É possível encontrar textos que se assemelhem a essa proposta. Como exemplo, um texto breve, de 1975, de William Cavanaugh [4] e, mais recentemente, o trabalho de Guilherme de Martini et. al. [6] e parte da dissertação de Juscelino de Oliveira [14] também se aproximam dessa proposta.

\footnotetext{
${ }^{1}$ Ponto de mudança de sentido de uma curva. No caso descrito pode ser visto como um ponto de distância máxima ao centro da circunferência externa.

${ }^{2}$ Segundo a Base Nacional Curricular Comum [13], aparecem nos anos finais do Ensino Fundamental, Unidade Temática "Números", Habilidade EF07MA01 e Unidade Temática "Álgebra", Habilidades EF07MA13, EF07MA17, EF08MA12, EF08MA13 e EF09MA07, e também no Ensino Médio, Componente Específica 5, Habilidade EM13MAT510. É curioso notar que os temas da proposta didática não aparecem na Unidade Didática "Geometria" apesar da interpretação geométrica possível.
} 


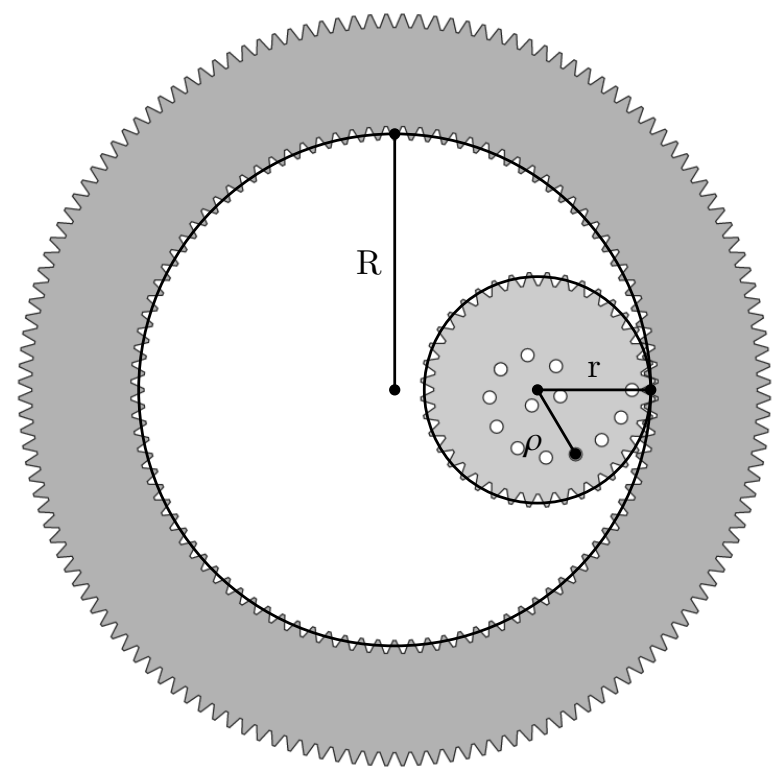

Figura 2: Representação de R, r e $\rho$ em um espirógrafo
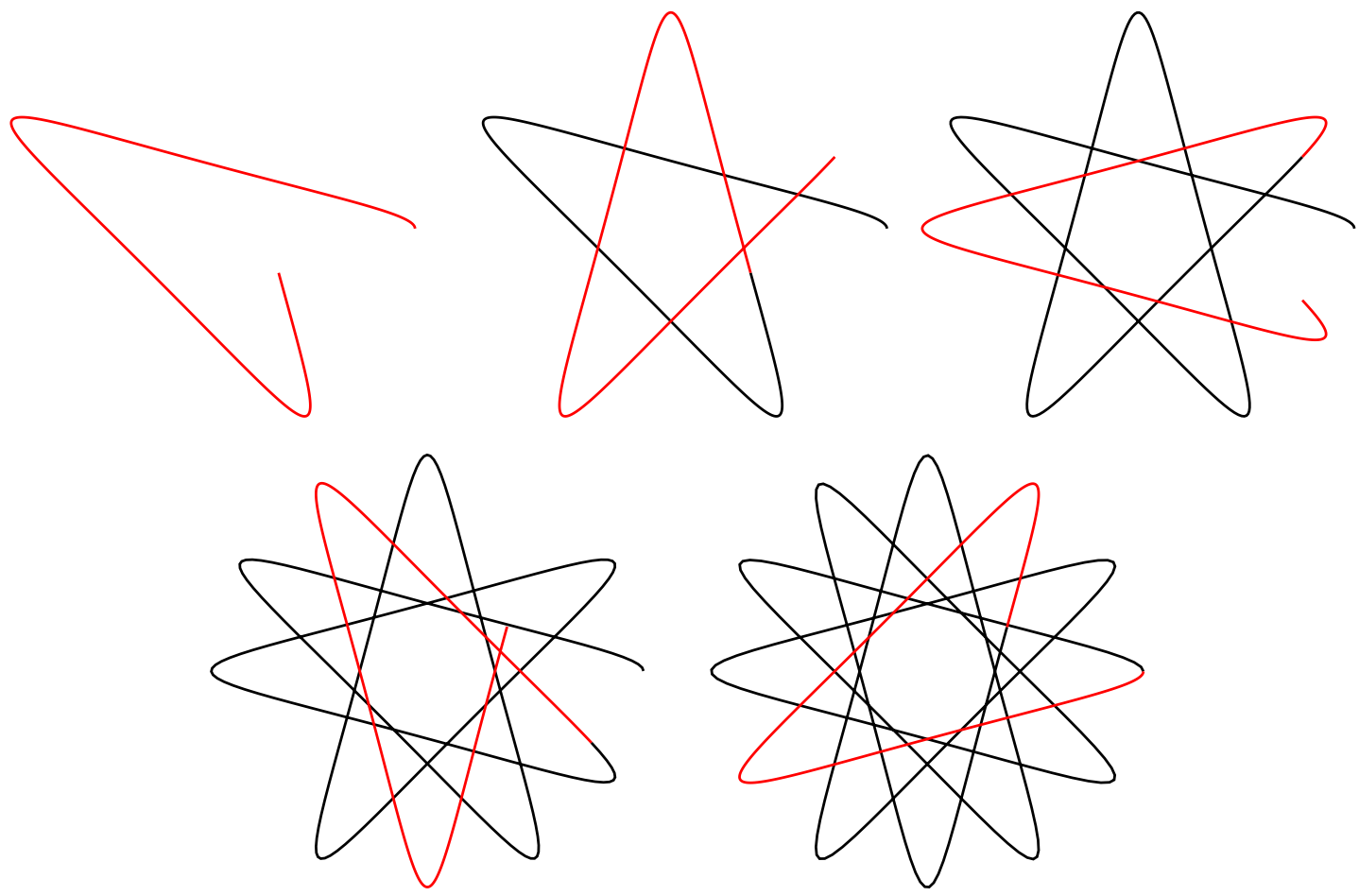

Figura 3: Diferentes estágios do desenho obtido pela configuração da Figura 2, a cada volta completa da engrenagem interna em torno da externa 
São consideradas as seguintes grandezas:

- Os números de dentes das engrenagens externa e interna utilizadas, denotados E e I, respectivamente.

- A escolha de um orifício da engrenagem interna e sua distância ao centro dessa engrenagem, denotada $\rho$.

- Os movimentos de ano (quando a engrenagem interna volta a tangenciar a externa no ponto de tangência original) e de dia (quando o ponto de tangência original da engrenagem interna volta a tangenciar engrenagem externa). Essas grandezas estão representadas na Figura 4.

- As distâncias $\mathrm{d}_{\max }$ e $\mathrm{d}_{\min }$ da hipotrocoide ao centro da engrenagem externa. Tais grandezas, bem como a coroa circular que encapsula o desenho, podem ser vistas em destaque na Figura 5.

No que se refere à capacidade de predição de características dos desenhos traçados pelo espirógrafo, os objetivos são:

1. Perceber que o número de dentes e o raio dos discos são proporcionais: $\mathrm{E} / \mathrm{R}=\mathrm{I} / \mathrm{r}$.

2. Determinar o número $\mathrm{A}$ de anos e o número $\mathrm{D}$ de dias até o desenho se completar como função de E e I.

3. Determinar as distâncias máxima $\left(\mathrm{d}_{\max }\right)$ e mínima $\left(\mathrm{d}_{\min }\right)$ da coroa circular que encapsula o desenho até o centro da engrenagem externa como função de R, r e $\rho$.

No que se refere ao objetivo 1 , o raio das engrenagens é proporcional ao número de dentes, com a constante de proporcionalidade $\kappa=1 / 2 \pi$, pois a unidade de medida é a distância entre dois dentes consecutivos (que pode ser convertida para outra unidade realizando uma medição e dividindo o valor obtido pelo número de dentes). Temos então que

$$
\mathrm{R}=\kappa \cdot \mathrm{E} \quad \mathrm{e} \quad \mathrm{r}=\kappa \cdot \mathrm{I}
$$

Para o objetivo 2, basta perceber que a cada ano completado são percorridos E dentes e à cada dia completado são percorridos I dentes. Então o número de dentes percorridos para um certo número de anos é A · E e para um certo número de dias é D · I. Quando o desenho completa-se, ocorre pela primeira vez $\mathrm{A} \cdot \mathrm{E}=\mathrm{D} \cdot \mathrm{I}$, ou seja, ocorre pela primeira vez a igualdade entre um múltiplo de E e um múltiplo de I. Portando, temos

$$
\mathrm{A} \cdot \mathrm{E}=\mathrm{D} \cdot \mathrm{I}=\operatorname{mmc}(\mathrm{E}, \mathrm{I})
$$

onde mmc(E, I) é o mínimo múltiplo comum de E e I. Usando o fato de que o produto de dois números é igual ao produto do seu mínimo múltiplo comum e seu máximo divisor comum, temos

$$
\mathrm{A} \cdot \mathrm{E}=\mathrm{D} \cdot \mathrm{I}=\frac{\mathrm{E} \cdot \mathrm{I}}{\operatorname{mdc}(\mathrm{E}, \mathrm{I})}
$$

onde mdc(E, I) é o máximo divisor comum de E e I. Concluímos então:

$$
\mathrm{A}=\frac{\mathrm{I}}{\operatorname{mdc}(\mathrm{E}, \mathrm{I})} \quad \text { e } \quad \mathrm{D}=\frac{\mathrm{E}}{\operatorname{mdc}(\mathrm{E}, \mathrm{I})}
$$




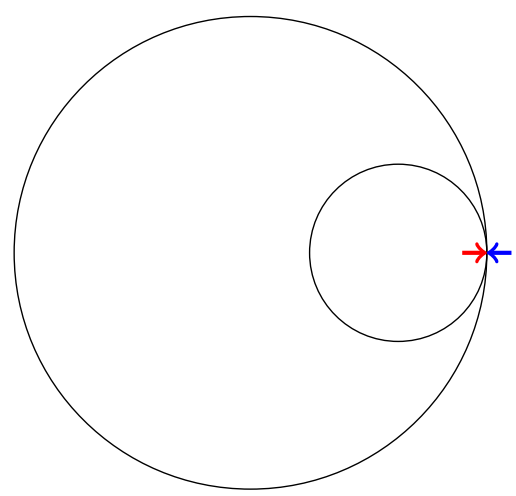

(a) Posição inicial: a seta vermelha indica o ponto inicial de tangência da circunferência interna com a externa, e a seta azul indica a posição inicial do movimento

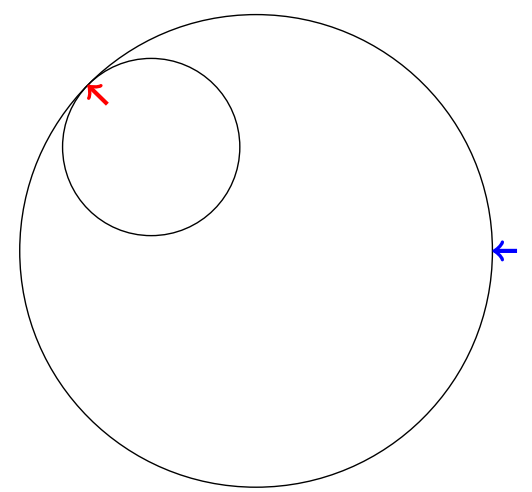

(b) Após completar 1 dia: a circunferência interna rolou até o ponto de tangência inicial voltar a tocar a circunferência externa

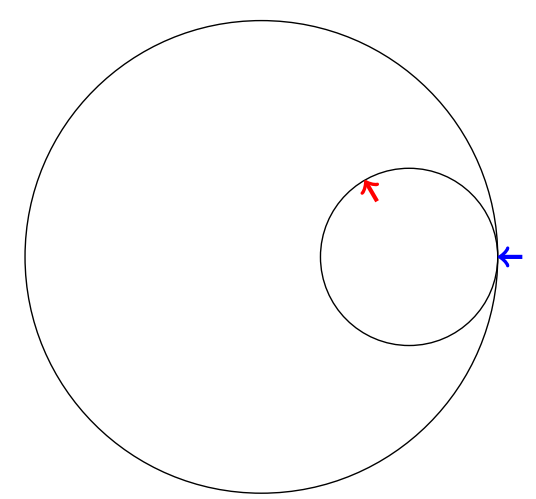

(c) Após completar 1 ano: a circunferência interna rolou até voltar a tocar a posição inicial do movimento

Figura 4: Representação de posições de um espirógrafo. Neste caso em particular, cada volta em torno da circunferência externa é igual a 2,67 voltas da interna em torno de si mesma

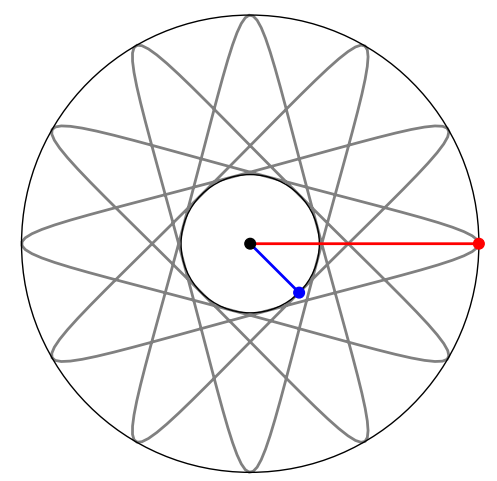

Figura 5: Desenho obtido pela configuração da Figura 2, com destaque para $\mathrm{d}_{\max }$ e $\mathrm{d}_{\min }$ 


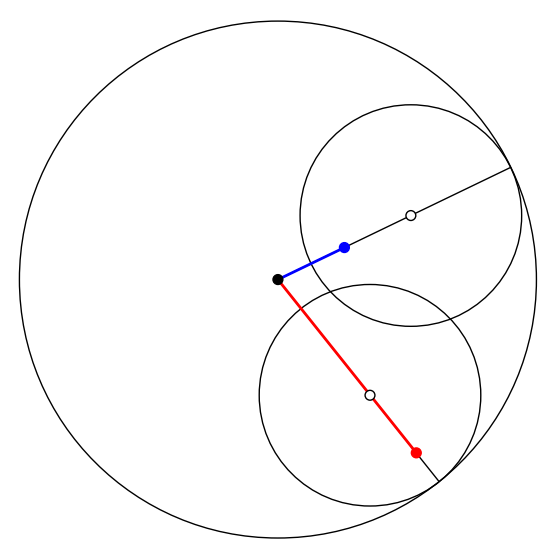

(a) $\mathrm{r}+\rho \leq \mathrm{R}$

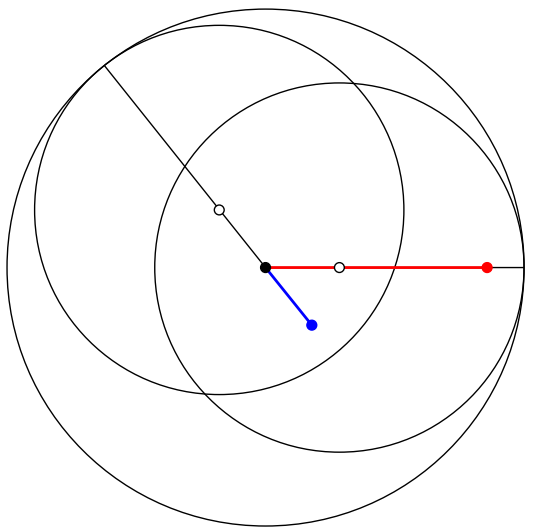

(b) $\mathrm{r}+\rho \geq \mathrm{R}$

Figura 6: Posições obtendo pontos de distância mínima e máxima consecutivos

Uma observação interessante é que, caso sejam usadas engrenagens com números de dentes iguais a um mesmo múltiplo m de $\mathrm{E}$ e I, isso não alterará $\mathrm{A}$ e $\mathrm{D}$, pois

$$
\frac{\mathrm{m} \cdot \mathrm{I}}{\mathrm{mdc}(\mathrm{m} \cdot \mathrm{E}, \mathrm{m} \cdot \mathrm{I})}=\frac{\mathrm{m} \cdot \mathrm{I}}{\mathrm{m} \cdot \mathrm{mdc}(\mathrm{E}, \mathrm{I})}=\frac{\mathrm{I}}{\operatorname{mdc}(\mathrm{E}, \mathrm{I})}
$$

e

$$
\frac{\mathrm{m} \cdot \mathrm{E}}{\mathrm{mdc}(\mathrm{m} \cdot \mathrm{E}, \mathrm{m} \cdot \mathrm{I})}=\frac{\mathrm{m} \cdot \mathrm{E}}{\mathrm{m} \cdot \mathrm{mdc}(\mathrm{E}, \mathrm{I})}=\frac{\mathrm{E}}{\operatorname{mdc}(\mathrm{E}, \mathrm{I})} .
$$

Para o objetivo 3 é conveniente olhar para as engrenagens em uma posição de distância máxima e uma de distância mínima e fazer o caminho entre o centro da engrenagem externa até o ponto desejado, passando por $\mathrm{R}, \mathrm{r}$ e $\rho$. A distância até um ponto de distância máxima sempre será

$$
\mathrm{d}_{\max }=\mathrm{R}-\mathrm{r}+\rho .
$$

Considerando a distância do centro da engrenagem externa até um ponto de distância mínima, duas possibilidades podem ocorrer, dependendo da soma de $\mathrm{r}+\rho$ :

$$
d_{\text {min }}=\left\{\begin{array}{lll}
\mathrm{R}-\mathrm{r}-\rho & \text { se } & \mathrm{r}+\rho \leq \mathrm{R} \\
\mathrm{R}+\mathrm{r}+\rho & \text { se } & \mathrm{r}+\rho \geq \mathrm{R} .
\end{array}\right.
$$

Ambas as possibilidades podem ser expressas por uma única fórmula:

$$
\mathrm{d}_{\min }=|\mathrm{R}-\mathrm{r}-\rho| .
$$

No caso da igualdade, $\mathrm{R}=\mathrm{r}+\rho, \mathrm{d}_{\min }=0$, e o desenho passa pelo centro da engrenagem externa. A Figura 6 representa os dois casos possíveis para $\mathrm{d}_{\min }$, com $\mathrm{r}+\rho \leq \mathrm{R}$ em 6 (a) e $\mathrm{r} \rho \geq \mathrm{R}$ em 6 (b).

Se $\mathbf{P} \in \mathbf{Z}$ é o número de dentes de uma engrenagem com raio igual a $\rho$ (distância do orifício utilizado para traçar a curva ao centro do disco móvel), então

$$
\rho=\kappa \cdot \mathrm{P} \text {. }
$$


Substituindo $\mathrm{R}=\kappa \mathrm{E}, \mathrm{r}=\kappa \mathrm{I}$ e $\rho=\kappa \mathrm{P}$ em (1) e (2) temos

$$
\mathrm{d}_{\max }=\kappa(\mathrm{E}-\mathrm{I}+\mathrm{P}) \quad \text { e } \quad \mathrm{d}_{\min }=\kappa|\mathrm{E}-\mathrm{I}-\mathrm{P}| .
$$

Fazendo $\mathrm{l}=\mathrm{P} / \mathrm{I}$ a razão entre $\rho$ e $\mathrm{r}$ e $\mathrm{k}=\mathrm{I} / \mathrm{E}$ a razão entre $\mathrm{r}$ e $\mathrm{R}$, obtemos as distâncias máxima relativa e mínima relativa:

$$
\mathrm{d}_{\max , \mathrm{rel}}=\kappa \mathrm{E}(1-\mathrm{k}(1+\mathrm{l})) \quad \text { e } \quad \mathrm{d}_{\min , \mathrm{rel}}=\kappa \mathrm{E}|1-\mathrm{k}(1+\mathrm{l})| .
$$

\section{Descoberta orientada e demonstração transformacional}

Esta seção trata da contextualização da sequência de atividades no que se refere ao método indutivo em geral e ao contexto de ensino de matemática em particular. Em seguida será comentada a estrutura geral da sequência, as características gerais do material que a compõe e as expectativas de ensino e aprendizagem, tanto especificamente, quanto como um modelo que pode ser adaptado para outras propostas.

Segundo Prince e Felder [15], um método indutivo de ensino e aprendizagem é um método centrado no estudante e essencialmente construtivista. O que caracteriza um método construtivista é a tentativa do estudante de fazer sentido da sua experiência e incorporar o novo conhecimento pelo filtro do seu conhecimento prévio. O papel do professor é então preparar experiências que favoreçam os estudantes à construírem o conhecimento por eles mesmos.

Os métodos indutivos classificados pelos autores, de acordo com suas características definidoras, são:

- Aprendizagem por investigação: Questões ou problemas proveem contexto para aprendizagem

- Aprendizagem baseada em problemas: Problemas complexos, com estrutura flexível, solução aberta e do mundo real proveem contexto para aprendizagem

- Aprendizagem baseada em projetos: Grandes projetos proveem contexto para aprendizagem

- Ensino baseado em casos: Estudos de casos proveem contexto para aprendizagem

- Aprendizagem por descoberta: Estudantes descobrem o conteúdo do curso por eles próprios

- Ensino "just-in-time": Estudantes completam e submetem exercícios conceituais; instrutores ajustam aulas de acordo com suas respostas

Outras características presentes nos métodos indutivos de ensino e aprendizagem, em maior ou menor grau, são: aprendizagem autodirigida, aprendizagem ativa e aprendizagem colaborativa/ cooperativa.

A sequência de atividades desenvolvidas neste trabalho pode ser entendida como aprendizagem por descoberta. Como a sequência provê um grau significativo de orientações aos alunos e a transição é sempre mediada por um professor, é pertinente designá-la como um instrumento para descoberta orientada. Vale a pena ressaltar que, na estrutura de cada uma das atividades da sequência e no encadeamento entre elas, é possível encontrar semelhanças com o descrito por Cabral em [3]. Mais detalhes na Seção 4, onde será apresentada a estrutura das atividades. 
Do ponto de vista dos objetivos, pretende-se orientar os alunos a fazerem uma série de constatações na direção de alguns padrões matemáticos que emergem do espirógrafo. Essa é a parte da descoberta. A intervenção do professor é necessária para que cada etapa desse processo se consolide de maneira conceitual, na forma de um pequeno corpo de proposições, cujo entendimento pode ser visto como uma demostração matemática.

O entendimento do sentido de demonstração vem de [10], onde Harel e Sowder descrevem diferentes esquemas de demonstrações. Segundo os autores, há três classificações básicas: demonstração por convicção externa, demonstração empírica e demonstração analítica. Demonstrações por convicção externa geralmente se baseiam na autoridade de um professor ou de um livro, mas também podem depender da aparência do argumento ou de sua manipulação simbólica, sem a atribuição de um significado. Demonstrações empíricas apoiam-se em exemplos ou casos particulares. A terceira categoria divide-se em duas subcategorias: demonstração analítica transformacional e axiomática.

No decorrer da sequência as atividades irão propiciar aos alunos descobertas empíricas e a produção de demonstrações analíticas transformacionais. Ou seja, os alunos irão formar conjecturas, de caráter preditivo, para resolver problemas propostos e posteriormente generalizar. Esse esquema de demonstração é baseado em um raciocínio dedutivo apresentado de modo explícito e rigoroso, mesmo que não seja formalizado em proposição, teorema etc., como é usual ocorrer em esquemas axiomáticos presentes nos livros de matemática universitária e nos artigos de pesquisa.

As proposições que os alunos terão demonstrado ao final das atividades poderiam ser enunciadas da seguinte maneira:

Proposição 1. Se E e I são os números de dentes das engrenagens externa e interna de uma configuração do espirógrafo, então os números $\mathrm{A}$ de anos e $\mathrm{D}$ de dias até o desenho se completar são dados por

$$
\mathrm{A}=\frac{\mathrm{I}}{\operatorname{mdc}(\mathrm{E}, \mathrm{I})} \quad e \quad \mathrm{D}=\frac{\mathrm{E}}{\operatorname{mdc}(\mathrm{E}, \mathrm{I})} .
$$

Proposição 2. Se $\mathrm{R}$ é a medida do raio da engrenagem externa do espirógrafo, $\mathrm{r}$ a medida do raio da engrenagem interna e $\rho$ a distância entre o centro da engrenagem interna e o orifício escolhido, então as medidas dos raios externo $\mathrm{d}_{\max }$ e interno $\mathrm{d}_{\min }$ da coroa circular que encapsula o desenho obtido são dadas por

$$
\mathrm{d}_{\text {max }}=\mathrm{R}-\mathrm{r}+\rho \quad e \quad \mathrm{~d}_{\min }=|\mathrm{R}-\mathrm{r}-\rho|
$$

\section{Proposta de sequência de atividades}

A Sequência de Atividades Didáticas (SAD) é composta de 11 atividades, quase todas com dois momentos distintos. O primeiro momento consiste de uma atividade a ser realizada em grupos pequenos de alunos (três a cinco) que começa sempre com uma manipulação do espirógrafo, uma orientação para uma medição ou uma observação de um fenômeno ${ }^{3}$. O segundo momento, que chamamos de sistematização, é quando a classe toda busca, junta, organizar, com mediação do professor, o que foi explorado no primeiro momento. É nesse segundo momento que ocorrem as demonstrações transformacionais, e ele também serve de ponto de partida para a atividade seguinte.

${ }^{3}$ Uma sugestão para o leitor interessado na organização de trabalhos em grupo em sala de aula é o livro de Elizabeth Cohen e Rachel Lotan [5]. 
Há um paralelo entre essa estrutura e o método hipotético-dedutivo que, como descrito em [9], consiste em:

i) Fazer observações.

ii) Formular uma hipótese que acomoda as observações.

iii) Deduzir novas predições observacionais da hipótese.

iv) Ver se as predições são verdadeiras. Se sim, voltar para iii). Caso contrário, tomar a hipótese como falseada e voltar para $i i)$.

Uma proposta semelhante de estruturação é apresentada em [3], onde vários paralelos com o presente trabalho podem ser encontrados. Do ponto de vista metodológico, a principal diferença entre esta $\mathrm{SAD}$ e a proposta por Cabral está no fato de que o ponto de partida, a questão motivadora que encontramos no seu texto, é um conteúdo matemático escolar (frações, funções etc.), enquanto a presente proposta parte de um objeto exógeno (espirógrafo) com propriedades matemáticas interessantes de serem exploradas.

Além das atividades diagramadas para uso direto pelos alunos, há um guia para o professor. $\mathrm{Na}$ elaboração do material do aluno, foi feito um esforço para que ele seja levado a formular por iniciativa própria as perguntas relevantes. A expectativa é que, com um modelo para sequências de atividades bem objetivado, como a presente proposta pretende alcançar, seja possível desenvolver outras sequências abordando outros temas matemáticos e outras experiências, utilizando materiais físicos ou digitais, seguindo um mesmo padrão.

Na continuação é exposto um panorama geral das atividades. Em seguida há uma apresentação mais detalhada de uma atividade em particular, a Atividade 6, explicitadas algumas considerações didáticas que nortearam a sua elaboração e redação.

A versão mais recente do material do aluno e do tutor poderão ser encontradas em < https://drive. google.com/drive/folders/1tdlrWqfKViP8sAtMaQNd85V_0KgAuh1t?usp=sharing >.

\subsection{Descrição das atividades}

A seguir serão descritas brevemente cada uma das 11 atividades que compõem a sequência.

\section{Atividade 1 - Primeiras observações}

A primeira atividade consiste em apresentar o espirógrafo aos alunos para que eles, através de observações, obtenham informações sobre o seu funcionamento. Nenhum desenho será feito ainda, mas os alunos deverão, pelas observações e respondendo algumas perguntas, imaginar como será o desenho obtido com o espirógrafo. Com isso eles terão um primeiro contato com o brinquedo e também com a elaboração de hipóteses, algo que percorrerá todas as atividades.

No momento de sistematização as informações obtidas pelos grupos e as suposições serão compartilhadas com a classe, e o professor ajudará na organização das ideias, separando as relevantes das irrelevantes para o estudo que seguirá e trazendo pontos pertinentes à discussão que eventualmente não tenham sido notados pelos alunos. Essa sistematização será posteriormente comparada com as novas sistematizações obtidas nas atividades seguintes. 
Síntese da aprendizagem: perceber os parâmetros relevantes no estudo discreto do espirógrafo.

\section{Atividade 2 - Primeiros desenhos}

Nesta atividade os alunos deverão fazer alguns desenhos livres com o espirógrafo para se familiarizarem com seu funcionamento e os desenhos que ele produz. Deve ser combinando entre os membros de cada grupo para que utilizem engrenagens diferentes, para uma maior variedade, mas ainda sem seguir um padrão. Com os desenhos prontos cada grupo irá listar semelhanças e diferenças. Com uma tentativa de descrição em palavras dos desenhos, essas semelhanças e diferenças aparecerão naturalmente.

Na sistematização o professor irá novamente separar as informações relevantes das irrelevantes para a proposta didática e ressaltar dois fatos:

1. Alguns desenhos tem mais "voltas" do que outros. Isso tem a ver com o número de anos e dias e irá compor a parte da SAD relacionada à Proposição 1.

2. Alguns desenhos são mais "estreitos" do que outros. Isso tem a ver com $\mathrm{d}_{\max }$ e $\mathrm{d}_{\min }$ e irá compor a parte da $\mathrm{SAD}$ relacionada à Proposição 2.

Ao final da sequência didática os alunos serão capazes de prever e controlar essas mudanças nos desenhos.

Síntese da aprendizagem: perceber tipos diferentes de desenhos que podem ser obtidos com o espirógrafo, estabelecendo semelhanças e diferenças entre eles.

\section{Atividade 3 - Nomeando os componentes}

Esta atividade não terá a parte dos grupos, sendo realizada apenas em conjunto com toda a sala. Os alunos deverão, com ajuda do professor, nomear cada componente do espirógrafo para facilitar a comunicação entre a sala nas atividades seguintes e também as formulações matemáticas subsequentes. A nomenclatura escolhida deve ser intuitiva e se relacionar com certos aspectos das peças, mas nesse momento não se espera q elas sejam definitivas. Haverá oportunidade de melhorar as nomenclaturas nas atividades seguintes.

\section{Atividade 4 - Mudando as configurações}

Aqui os alunos deverão, através de mudanças específicas de configurações de engrenagens e orifícios, notar qual a influência de cada mudança no desenho obtido. Ou seja, eles irão perceber que a escolha das engrenagens externa e interna altera o número de "voltas" do desenho, ou seja, o número de anos e dias, e que a escolha de orifícios diferentes para um mesmo par de engrenagens altera a "espessura" do desenho, ou seja, a diferença entre $d_{\max }$ e $d_{\min }$. Esses são os parâmetros que podem ser controlados.

Caso algum grupo não percebam algum desses fatos, isso será retomado na sistematização. É importante que essas mudanças nos desenhos estajam claras pois motivarão as próximas atividades. Uma vez estabelecido o que muda, a investigação será agora para responder como as mudanças acontecem, dependendo dos parâmetros escolhidos. 
Síntese da aprendizagem: perceber de forma qualitativa as mudanças no desenho de acordo com as mudanças nos parâmetros iniciais.

\section{Atividade 5 - Medições I}

Neste ponto os alunos começarão uma abordagem quantitativa dos desenhos. Será realizada uma contagem do número de dentes de cada engrenagem e uma medição do comprimento dos seus raios. As contagens devem ser diretas e as medições podem ser feitas de várias maneiras diferentes com a obtenção dos raios de maneira indireta. Vale ressaltar que a contagem será exata enquanto as medições fornecerão valores aproximados. Os valores serão preenchidos em uma tabela no material do estudante.

Na sistematização, os valores obtidos pelos grupos serão compartilhados com a classe para todos chegarem a um acordo e resolver eventuais discrepâncias. Os comprimentos dos raios das engrenagens serão acertados, por exemplo, tirando a média do valor obtido para cada engrenagem por cada grupo. A partir desse ponto todos irão usar os mesmos valores. Será um bom momento para atualizar os nomes dados às engrenagens para uma designação que envolve o número de dentes.

Síntese da aprendizagem: discretizar o espirógrafo, concluindo que o número de dentes de um disco é proporcional ao seu raio.

\section{Atividade 6 - anos e dias}

Esta atividade inicia uma abordagem sistemática para resolver o segundo objetivo da sequência. Os grupos serão apresentados ao que foi chamado de ano e dia e deverão contar esses valores para duas configurações diferentes de engrenagens, preenchendo os valores obtidos em tabelas apropriadas no material. Para facilitar a contagem, é sugerido usar uma caneta não permanente para indicar a posição inicial de contato das duas engrenagens.

Os valores obtidos pelos grupos serão reunidos em uma tabela única no momento de sistematização. Nessa tabela deverá constar todas as informações referentes a anos e dias das combinações de engrenagens utilizadas pelos grupos. O objetivo é encontrar um padrão. A maneira que os valores serão dispostos na tabela no material do aluno foi pensada para facilitar a visualização da relação existente entre os números de dentes das engrenagens em cada configuração e o número de anos e dias no desenho por ela produzida. Algumas combinações adicionais poderão ser estudadas nesse momento caso haja necessidade. Uma conjectura deve ser registrada ao final dessa sistematização.

Síntese da aprendizagem: perceber empiricamente que $\mathrm{A} \cdot \mathrm{E}=\mathrm{D} \cdot \mathrm{I}$.

\section{Atividade 7 - Relação entre número de dentes, anos e dias}

Nesta atividade os grupos irão primeiro calcular os produtos $\mathrm{A} \cdot \mathrm{E}=\mathrm{D} \cdot \mathrm{I}$ para as configurações que usaram na atividade anterior e depois generalizar. Irão depois responder o que representa esse valor obtido em relação a A, E, D e I.

Conhecendo esse produto, os alunos serão capazes de determinar o número de anos e dias antes de fazerem o desenho. 
Na sistematização será concluído, por todas as observações feitas até esse momento e com ajuda do professor, se necessário, que esse produto é o mínimo múltiplo comum dos números de dentes das engrenagens utilizadas.

Síntese da aprendizagem: concluir que $\operatorname{mmc}(\mathrm{E}, \mathrm{I})=\mathrm{A} \cdot \mathrm{E}=\mathrm{D} \cdot \mathrm{I}$.

\section{Atividade 8 - Testando a relação I}

Esta é a primeira atividade onde os grupos irão utilizar a fórmula obtida para prever aspectos do desenho sabendo apenas das engrenagens utilizadas. Eles deverão calcular A e D para uma configuração inédita, sabendo apenas sobre E e I. Depois farão o desenho para confirmar os valores previstos. Essa atividade concluirá o segundo objetivo da sequência.

Síntese da aprendizagem: verificação empírica da conclusão obtida na atividade anterior.

\section{Atividade 9 - Medições II}

Esta atividade inicia a abordagem para resolver o terceiro objetivo da sequência. Os alunos estarão agora interessados em como o orifício escolhido altera o desenho obtido com uma mesma configuração. Cada orifício está relacionado a um $\rho$, que também será medido pelos alunos nesse ponto. Isso será feito pela observação de desenhos resultantes de duas configurações diferentes de engrenagens e alguns orifícios diferentes em cada configuração. A diferença entre as configurações é que na primeira o raio da engrenagem externa é maior que o diâmetro da interna, e na segunda é menor. Os valores $\mathrm{d}_{\max }$ e $\mathrm{d}_{\min }$ serão medidos e registrados em tabela própria no material do aluno. Com esses valores, os alunos deverão notar algum padrão.

Na sistematização, duas configurações em particular, uma obedecendo cada citério, serão estudadas de maneira a obter uma tabela conjunta contendo os valores de $\mathrm{d}_{\max }$ e $\mathrm{d}_{\min }$ para todos os orifícios. Isso evidenciará o que ocorre com a mudança do orifício escolhido nos dois casos e também a particularidade do segundo caso com relação a $d_{\text {min }}$.

Síntese da aprendizagem: perceber que a mudança do orifício utilizado na engrenagem interna influencia na espessura da coroa circular que encapsula o desenho.

\section{Atividade 10 - Relação entre $\rho$ e os raios interno e externo}

Aqui os grupos irão fazer uma conjectura sobre como $d_{\max }$ e $d_{\min }$ são influenciados por $R, r$ e $\rho$ separadamente, inicialmente, para cada uma das configurações, e depois juntarão ambas as fórmulas em uma só que descreve o caso geral. A sugestão dada é observar o caminho entre o centro do desenho até um ponto de distância máxima e um de distância mínima percorrendo $\mathrm{R}, \mathrm{r}$ e $\rho$ em sequência.

$\mathrm{Na}$ sistematização as conclusões dos grupos serão discutidas para se chegar a um consenso sobre as fórmulas desejadas. O valor obtido por essas fórmulas será aproximado. Ao contrário de anos e dias que são grandezas discretas, as distâncias são grandezas contínuas, e por isso haverá o erro da medição. Mas um valor aproximado será suficiente.

Síntese da aprendizagem: concluir que $\mathrm{d}_{\max }=\mathrm{R}-\mathrm{r}+\rho$ e $\mathrm{d}_{\min }=|\mathrm{R}-\mathrm{r}-\rho|$. 


\section{anos e dias}

Iremos agora começar nosso estudo de uma forma mais estruturada. Em folha apropriada, complete dois desenhos usando uma mesma combinação de engrenagens, mas orificios diferentes da engrenagem interna. Conforme for desenhando, conte o número de voltas que a engrenagem interna dá em torno da engrenagem externa e depois o número de vezes que um ponto fixo da engrenagem interna toca a engrenagem externa. Juntamente com os desenhos, registre quais engrenagens e quais orificios foram usados.

Aqui é conveniente definir um ano como o movimento que a engrenagem interna faz até completar uma volta em torno da engrenagem extarna e um dia como o movimento que ela faz entre dois contatos consecutivos de um ponto fixo dela com a engrenagem externa.

Repita este processo com outra configuaçāo de engrenagens, completando quatro desenhos no total. Registre os resultados dessa atividade na tabela abaixo. Como sugestão, para a Configuração 1 use engrenagens tal que o número de dentes da engrenagem externa seja maior que o dobro do número de dentes da interna e para a Configuração 2 use engrenagem tal que esse número seja menor que o dobro do número de dentes da engrenagem externa.

\begin{tabular}{|l|l|}
\hline \multicolumn{2}{|c|}{ Configuração 1} \\
\hline Engrenagem externa & \\
\hline Engrenagem interna & \\
\hline Orificio \\
\hline \# anos \\
\hline$\#$ dias \\
\hline \hline \multicolumn{2}{|c|}{ Configuração 2} \\
\hline Engrenagem externa & \\
\hline Engrenagem interna & \\
\hline Orificio \\
\hline \# anos \\
\hline$\#$ dias \\
\hline
\end{tabular}

\section{Sistematização da aprendizagem}

Iremos agora juntar todas as informaçōes obtidas pela sala e buscar por padröes. Nesta sistematizaçăo, concluiremos primeiramente o que muda no desenho com cada mudança de configuração que fazemos com as engrenagens e os orificios.

Depois, construiremos uma tabela conjunta com as informações obtidas por cada grupo nessa atividade. Começando com uma escolha do professor, a tabela irá conter os seguintes itens nas suas colunas, em ordem: $N$ (Número do grupo). $E$ (dentes na engrenagem externa), $A$ (número de anos), I (dentes na engrenagem interna) e $D$ (número de dias). Observando a tabela nessa disposiçăo, que iremos notar? Registre nesta página todas as conclusões obtidas pela turma.

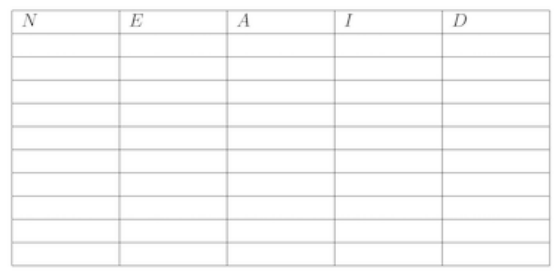

Espaço para anotar as conclusões:

Figura 7: Reprodução da Atividade 6 do material do aluno

\section{Atividade 11 - Testando a relação II}

Novamente os grupos irão utilizar fórmulas obtidas na atividade anterior para fazerem previsões. Escolhendo duas configurações seguindo os critérios estabelecidos anteriormente quanto ao raio da engrenagem externa e o diâmetro da interna, os alunos deverão calcular $d_{\max }$ e $d_{\min }$ para cada configuração para um $\rho$ em particular, registrando os valores obtidos em tabela própria no material. Depois farão os desenhos para confirmar os valores previstos, levando em conta o fato de que são aproximados. Aqui se completará o terceiro objetivo.

Síntese da aprendizagem: verificação empírica da conclusão obtida na atividade anterior.

\subsection{Atividade 6}

O desenvolvimento dessas atividades, elaboradas pensando em outorgar um alto grau de autonomia aos alunos, foi pautado por muitos dilemas e demandou grande atenção a detalhes importantes. Para ilustrar os diversos dilemas envolvidos, na busca de um equilíbrio que permita uma descoberta genuína e factível, a seguir é apresentada em detalhes a Atividade 6, reproduzida na Figura 7 conforme aparece no material a ser distribuído aos alunos.

O ponto de partida dessa atividade é a sistematização da anterior, na qual foi evidenciado que o número de dentes de uma engrenagem é proporcional ao seu raio. Na Atividade 5 foi estabelecida a notação básica: E e I, número de dentes da engrenagem externa e interna respectivamente, e A e D, número de anos e dias transcorridos até se obter uma figura fechada. O objetivo dessa 


\begin{tabular}{|l|l|l|l|}
\hline E & A & I & D \\
\hline 96 & 1 & 32 & 3 \\
\hline 105 & 3 & 45 & 7 \\
\hline
\end{tabular}

Tabela 1: Exemplo de sugestão do professor para valores de E, A, I e D.

atividade é a constatação de que $\mathrm{A} \cdot \mathrm{E}=\mathrm{D} \cdot \mathrm{I}$. Vale mencionar que essa sistematização será o ponto de partida da atividade seguinte.

Os alunos serão orientados a realizar dois pares de desenhos, cada um com uma configuração diferente de engrenagens e, dentro de cada par, orifícios diferentes da engrenagem interna. O primeiro fato notável é que o número de anos e de dias não depende do orifício escolhido, então deve depender apenas do número de dentes das engrenagens escolhidas.

Juntando os dados obtidos pelos grupos será montada, na sistematização, uma tabela onde nas colunas constam, nesta ordem: o número de dentes da engrenagem externa, o número de anos até o desenho se completar, o número de dentes da engrenagem interna e o número de dias até o desenho se completar. As colunas da tabela foram ordenadas propositalmente, de modo a termos os fatores E, A e I, D adjacentes, para facilitar a visualização da relação E · A = I · D.

É importante destacar que, a depender das escolhas das engrenagens, o número de dias e anos e os produtos $\mathrm{E} \cdot \mathrm{A}$ e I $\cdot \mathrm{D}$ podem assumir valores elevados, de modo que a relação de igualdade não seja evidenciada pelos alunos. Nesse sentido, caso seja necessário, o professor pode sugerir alguns pares adicionais de engrenagens, de modo a obter, por exemplo, as linhas da Tabela 1.

Pensando em uma sala de tamanho típico, é razoável supor que a tabela dessa atividade tenha de 20 a 30 linhas. Evidenciar a igualdade $\mathrm{E} \cdot \mathrm{A}=\mathrm{I} \cdot \mathrm{D}$ torna-se assim algo relativamente laborioso. No caderno de orientações ao professor, sugerimos duas estratégias possíveis: compartilhar a tarefa entre os alunos (cada grupo faz as contas relativas a suas medições) ou fazer as anotações diretamente em uma planilha digital, projetada no quadro.

Perceber a regularidade dessa igualdade levará à sistematização da Atividade 6, que será o ponto de partida da atividade seguinte, na qual se pretende notar que o produto $\mathrm{E} \cdot \mathrm{A}$ (ou I $\cdot \mathrm{D}$ ) é o mínimo múltiplo comum dos números de dentes das engrenagens usadas, E e I.

\section{Considerações Finais}

A sequência de atividades didáticas apresentadas neste trabalho consiste de tarefas bastante detalhadas para serem oferecidas aos alunos, com o intuito de aguçar o seu olhar e orientar a sua descoberta. Esse detalhamento, assim como as sugestões e orientações aos professores, visam facilitar o seu trabalho, mas de modo algum substituí-lo. Pelo contrário, para que esse tipo de atividade possa atingir seus objetivos, um pressuposto básico é a participação ativa de um professor matematicamente competente e pedagogicamente sensível.

Devido à pandemia de Covid-19 e às restrições de prazo de pós-graduação, tais atividade não foram testadas, como era a intenção dos autores. Se você for um desses professores competente e sensível a utilizar essas atividades, nós, os autores, ficaremos muito gratos em receber o seu retorno, seus comentários, correções, sugestões e críticas. 


\section{Referências}

[1] Isto é Matemática, Sociedade Portuguesa de Matemática, Disponível em <https://www.spm.pt/ istoematematica/>, Acesso em: 3 de fevereiro de 2022.

[2] Toy, Spirograph. NATIONAL MUSEUM OF AMERICAN HISTORY. Disponível em < https: //americanhistory.si.edu/collections/search/object/nmah_1292825>. Acesso em: 8 de maio de 2021.

[3] Cabral, N. F. Sequências Didáticas. Belém, SBEM / SBEM-PA, 2017.

[4] Cavanaugh, W. E. "The Spirograph and the Greatest Commom Factor". The Mathematics Teacher, v. 68, $\mathrm{n}^{\circ} 2$, p. 162-163, 1975.

[5] Cohen, E. G; Lotan, R. A. Planejando o trabalho em grupo: estratégias para salas de aula heterogêneas. Porto Alegre, Penso Editora, 2017.

[6] De Martini, G; Chang, P; Michelson, L. L; Mendonça, S. C; Vertuan, R. E. Modelagem Matemática com Desenhos Construídos pela Régua Mágica. IV ENDICT - Encontro de Iniciação Científica, Universidade Tecnológica Federal do Paraná, Toledo, 2016.

[7] Deck, K. M. "Spirograph Math". Humanistic Mathematics Network Journal, \#19, p. 13-17, 1999.

[8] Nathan Friend, Inspiral Web, Disponível em <https://nathanfriend.io/inspiral-web/>, Acesso em: 3 de fevereiro de 2022.

[9] Godfrey-Smith, P. Theory and Reality. Chicago, The University of Chicago Press, 2003.

[10] Harel, G; Sowder, L. "Students' Proof Schemes: Results from Exploratory Studies". CBMS Issues in Mathematics Education, v.7, p. 234-283, 1998.

[11] Jesus, F. G. Espirógrafo, hipotrocoides e epitrocoides : atividades exploratórias de curvas planas para o ensino médio utilizando o GeoGebra. Dissertação (mestrado profissional) - Universidade Federal de Mato Grosso, Instituto de Ciências Exatas e da Terra, Programa de PósGraduação Profissional em Matemática, Pontal do Araguaia, 2019.

[12] Lockwood, E. H. A Book of Curves. Cambridge, Cambridge University Press, 1961.

[13] Ministério da Educação Base Nacional Curricular Comum. Brasília, 2018.

[14] Oliveira, J. W. Curvas Planas Parametrizadas, Curvas Cicloidais e Espirógrafo. Faculdade de Ciências da Universidade do Porto, Departamento de Matemática, Mestrado em Matemática para Professores, Portugal, 2018.

[15] Prince, M. J; Felder, R. M. "Inductive Teaching and Learning Methods: Definitions, Comparisions and Research Bases". Journal of Engineering Education, v. 95, n² 2, p. 123-138, 2006.

[16] Pólya, G. How to Solve It. Princeton e Oxford, Princeton University Press, 2004.

[17] Weisstein, E. W. Roulette. MathWorld - A Wolfram Web Resource. Disponível em: <https: //mathworld.wolfram.com/Roulette.html>. Acesso em: 8 de maio de 2021. 
Fernando de Oliveira Cezarino Universidade Estadual de Campinas $<$ f085855@dac.unicamp.br>

Marcelo Firer Universidade Estadual de Campinas <mfirer@ime.unicamp.br>

Recebido: $12 / 11 / 2021$

Publicado: 04/03/2022 